\title{
Evaluation of a recombinant antigen ELISA for the diagnosis of acute toxoplasmosis and comparison with traditional antigen ELISAs
}

\author{
A. M. JOHNSON*, HELEN ROBERTS and ASTRID M. TENTER $\dagger$
}

Department of Microbiology and Infectious Diseases, Flinders University and Flinders Medical Centre, Bedford Park, South Australia 5042

\begin{abstract}
Summary. An evaluation of five ELISA methods for the diagnosis of acute toxoplasmosis was undertaken by comparing a laboratory-produced ELISA employing two recombinant Toxoplasma gondii polypeptides as antigen with a laboratory-produced ELISA and three commercially available ELISAs employing traditional parasite antigen preparations derived from whole tachyzoites. With a panel of 75 sera from patients who showed either serological and clinical evidence of acute toxoplasmosis, or of diseases caused by other infectious agents, or from patients who showed no evidence of infectious disease, the ELISAs gave overall positive predictive values of $81.6-100 \%$, negative predictive values of $87.8-100 \%$, sensitivities of $81 \cdot 3-100 \%$, and specificities of $83 \cdot 7-100 \%$. No two ELISAs gave identical results with all sera tested. In total, the ELISA based on the two recombinant $T$. gondii polypeptides appeared to be the most specific ELISA in this comparison, showing positive predictive values and specificities of $100 \%$ for all groups of patients tested. The overall negative predictive value for this ELISA was $87.8 \%$ and the sensitivity was $81.3 \%$. Therefore, the ELISA based on recombinant antigen appears to be a promising advance in the serological diagnosis of acute toxoplasmosis.
\end{abstract}

\section{Introduction}

Toxoplasmosis may cause abortion or fetal abnormalities if first contracted during pregnancy, but exposure to the parasite is usually accompanied by very mild symptoms, if any, in the mother. ${ }^{1}$ Therefore, much research has been performed to diagnose acute toxoplasmosis. Consequently, tests for the detection of Toxoplasma gondii-specific IgM have become commercially available and are used extensively in diagnostic laboratories.

Because of its ease of use, cost-effectiveness and high sensitivity and specificity, the ELISA has replaced older tests such as indirect immunofluorescence, complement fixation, and indirect haemagglutination in many laboratories. ${ }^{2}$ However, discrepancies have been noted among the results obtained with several commercially available ELISAs for the diagnosis of acute toxoplasmosis. ${ }^{3-7}$

These commercial ELISAs are usually based on antigen preparations derived from tachyzoites of the

Received 14 Oct. 1991; revised version accepted 20 March 1992.

* Present address: Department of Microbiology, School of Biological and Biomedical Sciences, University of Technology Sydney, Westbourne Street, Gore Hill NSW, Australia 2065.

$\dagger$ Present address: Institut für Parasitologie, Tierärztlichen Hochschule Hannover, Bünteweg 17, W-3000 Hannover 71, Germany.
RH strain of $T$. gondii. This stage of the parasite is obligately intracellular and hence must be grown in tissue culture or the peritoneal cavities of mice. Consequently, the ELISA antigens prepared by the traditional method are not composed of defined parasite antigens and are inevitably contaminated with at least some host tissue. We have recently cloned and sequenced two $T$. gondii gene fragments, ${ }^{8}$ termed $\mathrm{H} 4$ and H11, the glutathione-S transferase (GST) fusion polypeptides of which can be used for the diagnosis of acute toxoplasmosis in experimentally infected mice $^{9}$ and naturally infected cats, ${ }^{10}$ sheep ${ }^{10}$ and man. ${ }^{11}$ The aims of the study reported here were to compare an ELISA based upon the two recombinant $T$. gondii fusion polypeptides (H4/GST and H11/GST) as antigen (REC-ELISA) with a laboratory-produced antibody-class capture ${ }^{12,13}$ ELISA (FMC-ELISA) and three commercially available ELISAs for the diagnosis of acute toxoplasmosis.

\section{Materials and methods}

\section{ELISAs}

The distributors of five ELISAs for the detection of $T$. gondii-specific IgM that were commercially available in Australia were approached regarding the inclusion of their ELISA in the survey, and those 
included in the study were: Abbott Laboratories Diagnostics Division, Abbott Park, IL, USA, batch number 47887M100, expiry date 19 June 1991; Mercia Diagnostics Limited, Broadford Park, Shalford, Surrey, batch number 42, expiry date 11 Jan. 1991; and Sorin Biomedical Diagnostic Division, 13040 Saluggia, Vercelli, Italy, batch number 2350690A, expiry date 3 Oct. 1991.

The Abbott-ELISA was performed on an IMx automated analyser and this ELISA was supervised by staff of Abbott Australia. The other two commercial ELISAs were performed in the microtitration plates supplied by the manufacturers. These ELISAs were performed exactly according to the individual manufacturer's accompanying instructions. The methodology of the Abbott-ELISA IMx system, the MerciaELISA, the FMC-ELISA and the REC-ELISA, have all been described elsewhere., ${ }^{4,11,13}$ However, in the study reported here, the REC-ELISA antigen was an equal mixture of H4/GST and H11/GST, not separate H4/GST or H11/GST as used previously. Both the Abbott-ELISA and REC-ELISA are indirect ELISAs, the FMC-ELISA and Sorin-ELISA are antibody capture ELISAs with unlabelled antigen, and the Mercia-ELISA is an antibody capture ELISA with labelled antigen.

\section{Serum panel}

Sera were from four groups of patients.

Group 1-31 sera from 20 patients known to have acute toxoplasmosis with positive results for $T$. gondiispecific IgM in the FMC-ELISA. Six samples were sequential bleeds from one patient, ${ }^{14} 12$ sera were paired sera (about 14 days apart) from six patients, ${ }^{14}$ eight sera were single samples from eight patients, ${ }^{14}$ and five sera were provided by an Austrian and a French laboratory. ${ }^{13}$

Group 2-15 sera from 13 patients who had no previous history of exposure to $T$. gondii and were seronegative for both $T$. gondii-specific IgG and IgM in the FMC-ELISA for IgM and a standard ELISA for IgG. ${ }^{14}$

Group 3-15 sera from 15 patients who had no previous history of exposure to $T$. gondii and were seronegative for both $T$. gondii-specific IgG and IgM in the FMC-ELISA for IgM and a standard ELISA for IgG, ${ }^{14}$ but who possessed antibodies to rheumatoid factor or antinuclear factor (RF/ANF) or one of the following infectious agents: Epstein-Barr virus, Toxocara sp., Trichinella sp., cytomegalovirus or Mycoplasma pneumoniae.

Group 4-14 sera from 12 patients with previous discrepant recordings of $T$. gondii-specific IgM. Four of these sera were from three patients at the Flinders Medical Centre and the remaining 10 sera were from nine patients who had been found to give discrepant results when tested with the Mercia-ELISA, the SorinELISA, and an immunosorbent agglutination test for T. gondii-specific IgM by Mr D. Dickeson, Westmead Hospital, New South Wales.

\section{Precision}

To test the within-assay precision of the ELISAs, four sera (one with an OD $<0.2$, one with $0.2<\mathrm{OD}<$ 0.6 , one with $0.6<\mathrm{OD}<1.0$ and one with $\mathrm{OD}>1.0$ ) were tested nine times in one assay. To test the between-assay precision of the ELISAs, one serum with an OD of $>1.0$ was tested in at least three assays for each ELISA.

\section{Convenience of use}

As these kits were being evaluated for their convenience of use in the routine serological diagnosis of acute toxoplasmosis, the operator made a subjective assessment of each ELISA on a scale of 1 (least convenient) to 5 (most convenient) based on 12 criteria.

\section{Rheumatoid factor screening}

Sera were tested for the presence of rheumatoid factor (RF) with a commercial kit (Rapi Tex RF New) according to the manufacturer's instructions (Behringwerke AG, Marburg, Germany).

\section{Statistical analyses}

Sensitivity, specificity and positive and negative predictive values were calculated as outlined by Griner et al.$^{15}$ according to the following formulae:

$$
\begin{gathered}
\text { Sensitivity }=\frac{\text { true positives }}{\text { true positives }+ \text { false negatives; }} \\
\text { Specificity }=\frac{\text { true negatives }}{\text { false positives }+ \text { true negatives; }} \\
\text { Positive predictive value }= \\
\text { true positives } \\
\text { Negative predictive value }=
\end{gathered}
$$

$$
\frac{\text { true negatives }}{\text { false negatives }+ \text { true negatives. }}
$$

The definitions are as follows: sensitivity, the probability that the assay will be positive when the disease is present; specificity, the probability that the assay will be negative when the disease is not present; positive predictive value, the probability that the disease is present when the assay is positive; negative predictive value, the probability that the disease is not present when the assay is negative. As predictive values depend on the pre-test likelihood of disease, ${ }^{15}$ i.e., the prevalence of $T$. gondii-specific antibodies in a certain population, we evaluated the five ELISAs by comparing these values for each single group of patients tested as well as for the total panel of the 75 sera. 
Table I. Sensitivities, specificities and positive and negative predictive values of five ELISAs for the diagnosis of acute toxoplasmosis

\begin{tabular}{|c|c|c|c|c|c|c|c|c|c|c|c|c|c|}
\hline \multirow{3}{*}{ ELISA } & \multicolumn{2}{|c|}{ Sensitivity* (\%) } & \multicolumn{4}{|c|}{ Specificity $\dagger(\%)$} & \multirow{2}{*}{\multicolumn{3}{|c|}{$\begin{array}{c}\text { Positive } \\
\text { predictive } \\
\text { valueł }(\%)\end{array}$}} & \multirow{2}{*}{\multicolumn{4}{|c|}{$\begin{array}{c}\text { Negative } \\
\text { predictive } \\
\text { value† (\%) }\end{array}$}} \\
\hline & \multirow[t]{2}{*}{ Gpl } & \multirow[t]{2}{*}{ All } & \multirow[t]{2}{*}{ Gp2 } & \multirow[t]{2}{*}{ Gp3 } & \multirow[t]{2}{*}{ Gp4 } & \multirow[t]{2}{*}{ All } & & & & & & & \\
\hline & & & & & & & Gp1 & Gp4 & All & Gp2 & Gp3 & Gp4 & All \\
\hline Abbott & 96.8 & 96.9 & 100 & $86 \cdot 7$ & $61 \cdot 5$ & $83 \cdot 7$ & 100 & 16.7 & 81.6 & 100 & 100 & 100 & 97.3 \\
\hline Mercia & 100 & 100 & 100 & 100 & $84 \cdot 6$ & $95 \cdot 3$ & 100 & $33 \cdot 3$ & $94 \cdot 1$ & 100 & 100 & 100 & 100 \\
\hline Sorin & 96.8 & 93.8 & 100 & 100 & $84 \cdot 6$ & $95 \cdot 3$ & 100 & $\ldots \S$ & $93 \cdot 8$ & 100 & 100 & 91.7 & 95.3 \\
\hline FMC & 100 & 96.9 & 100 & 100 & 100 & 100 & 100 & $\ldots \S$ & 100 & 100 & 100 & 92.9 & $97 \cdot 7$ \\
\hline REC & 80.6 & $81 \cdot 3$ & 100 & 100 & 100 & 100 & 100 & 100 & 100 & 100 & 100 & 100 & 87.8 \\
\hline
\end{tabular}

*Sensitivity was not calculated separately for Groups $2-4$ because there was no $T$. gondii-positive serum in Groups 2 and 3, and only one T. gondii-positive serum in Group 4.

† Specificity and negative predictive value were not calculated separately for Group 1 because there was no $T$. gondii-negative serum in this group.

$\ddagger$ Positive predictive value was not calculated for Groups 2 and 3, because there was no $T$. gondii-positive serum in these groups.

$\S$ Positive predictive value could not be calculated accurately because the number of sera was too low.

\section{Results}

\section{Positive sera - Group 1}

One commercial ELISA, the Mercia-ELISA gave total agreement with the FMC-ELISA for the $31 T$. gondii-specific IgM-positive sera in this group. The Sorin-ELISA gave one false negative result, the Abbott-ELISA gave a false negative result on another serum sample, and the REC-ELISA gave false negative results on another six of the 31 sera tested. The calculated sensitivities and positive predictive values for this group are given in table I.

\section{Negative sera-Group 2}

This group of sera would appear to be the simplest to test as they did not contain antibodies to $T$. gondii nor to other major infectious agents. Not surprisingly therefore, all ELISAs gave specificities and negative predictive values of $100 \%$ for this group of sera (table I).

Table II. Results of five ELISAs for the 14 discrepant sera in Group 4

\begin{tabular}{c|ccccc}
\hline \multirow{2}{*}{$\begin{array}{c}\text { Specimen } \\
\text { no. }\end{array}$} & \multicolumn{5}{|c}{ ELISA result } \\
\cline { 2 - 6 } & Abbott & Mercia & Sorin & FMC & REC \\
\hline $1^{*}$ & + & + & - & - & - \\
$2^{*}$ & + & + & - & - & + \\
$3^{*}$ & - & + & - & - & - \\
4 & - & - & - & - & - \\
5 & - & - & - & - & - \\
6 & + & - & - & - & - \\
7 & - & - & - & - & - \\
$8^{*}$ & + & - & - & - & - \\
$9^{*}$ & - & - & + & - & - \\
10 & - & - & - & - & - \\
11 & + & - & - & - & - \\
12 & - & - & - & - & - \\
$13^{*}$ & - & - & + & - & - \\
$14^{*}$ & + & - & - & - & - \\
\hline
\end{tabular}

*Positive for RF.
Negative sera, positive for other infectious agents - Group 3

All of the ELISAs gave negative predictive values of $100 \%$ with this group of sera which did not have $T$. gondii-specific IgG of IgM, but did have antibodies to $\mathrm{RF} / \mathrm{ANF}$ or to one of a range of infectious agents. The Abbott-ELISA gave false positive results with one serum that was positive for $M$. pneumoniae and a serum that was positive for RF/ANF, giving a specificity of $86.7 \%$ (table I). Both of these sera still gave positive results for $T$. gondii-specific IgM with this ELISA even after being treated for rheumatoid factor as described by the manufacturer.

\section{Discrepant sera-Group 4}

Perhaps not surprisingly, this group of sera gave the most discordant results. Seven of the sera were found to contain RF, but this did not appear to correlate with false positive results (table II). The calculated specificities as well as positive and negative predictive values for this group of sera are listed in table I. The majority of ELISAs found serum no. 2 to be positive for $T$. gondii-specific IgM and the remainder to be negative (table II). These results suggest that serum no. 2 would indeed be positive for $T$. gondii-specific IgM.

To calculate the overall sensitivities, specificities, and positive and negative predictive values for the total panel of all 75 sera, 32 sera (31 sera of Group 1, one serum of Group 4) were defined as $T$. gondiipositive sera, and 43 sera (15 sera of Group 2, 15 sera of Group 3,13 sera of Group 4) were defined as $T$. gondii-negative sera. The results are contained in table I.

\section{Precision}

From the results of the four sera used in the withinassay analysis it appeared that generally the greater the amount of $T$. gondii-specific IgM in a serum, the smaller the coefficient of variation of the ELISA. The 
Table III. Comparison of coefficients of variation of five ELISAs for the diagnosis of acute toxoplasmosis on four different sera tested nine times in one assay

\begin{tabular}{|c|c|c|c|c|}
\hline \multirow{2}{*}{ ELISA } & \multicolumn{4}{|c|}{ Coefficient of variation (\%) of serum no. } \\
\hline & $\begin{array}{c}1 \\
(\mathrm{OD}<0 \cdot 2)\end{array}$ & $\stackrel{2}{2}(0.2<O D<0.6)$ & $(0.6<\mathrm{OD}<1.0)$ & $(\mathrm{OD}>1.0)$ \\
\hline $\begin{array}{l}\text { Abbott } \\
\text { FMC } \\
\text { Mercia } \\
\text { Sorin } \\
\text { REC }\end{array}$ & $\begin{array}{l}7 \cdot 22 \\
8 \cdot 22 \\
7 \cdot 31 \\
8.00 \\
\text { ND }\end{array}$ & $\begin{array}{r}3 \cdot 72 \\
5 \cdot 50 \\
\text { ND } \\
6 \cdot 11 \\
18.99\end{array}$ & $\begin{array}{r}2.87 \\
4.35 \\
3 \cdot 51 \\
4.74 \\
15.04\end{array}$ & $\begin{array}{l}2 \cdot 78 \\
8.08 \\
6 \cdot 63 \\
4.43 \\
4 \cdot 28\end{array}$ \\
\hline
\end{tabular}

ND, not done.

Table IV. Convenience of use of five ELISAs for the diagnosis of acute toxoplasmosis

\begin{tabular}{l|rrrrr}
\hline \multirow{2}{*}{ Parameter } & \multicolumn{5}{|c}{ ELISA } \\
\cline { 2 - 7 } & Abbott & Mercia & Sorin & FMC & REC \\
\hline Number of serum dilution steps & 0 & 1 & 1 & 1 & 1 \\
Serum dilution & 40 & 101 & 101 & 64 & 50 \\
Controls treated the same as tests & Yes & No & No & Yes & Yes \\
Incubation time (h) & $0 \cdot 45$ & $2 \cdot 5$ & $2 \cdot 5$ & $4 \cdot 5$ & $3 \cdot 16$ \\
Specimen type & Serum & Serum & Serum, & Serum, & Serum, \\
& & & plasma & plasma & plasma \\
Minimum sample volume used ( $\mu$ l) & 150 & 10 & 10 & 10 & 10 \\
Number of washes & NA & 5 & 5 & 3 & 4 \\
Wavelength for reading (nm) & NA & 450 & 450 & 405 & 405 \\
Clarity of written instructions & 3 & 4 & 3 & NA & NA \\
Presentation of reagents & 5 & 5 & 4 & NA & NA \\
Protocol for removal of RF & Yes & No & No & NA & NA \\
Expression and interpretation of results & 5 & 4 & 2 & 4 & 4 \\
Overall assessment & 5 & 4 & 3 & 3 & 3 \\
& & & & & \\
\hline
\end{tabular}

NA, not applicable.

coefficients of variation for the four sera for each ELISA are shown in table III. The between-assay analysis of precision gave coefficients of variation as follows: Abbott-ELISA, 4.86\% ; Sorin-ELISA, $8.1 \%$; Mercia-ELISA, $8.0 \%$; FMC-ELISA, $7.8 \%$; RECELISA, $3 \cdot 54 \%$.

\section{Convenience of use}

The assessment of convenience of use of each ELISA is given in table IV.

\section{Discussion}

Several recent studies ${ }^{4-7}$ have been performed to compare commercially available ELISAs for the detection of $T$.gondii-specific IgM, but few have included as many different ELISAs as compared here, nor have any of those studies involved an analysis of ELISAs based upon recombinant parasite fusion polypeptides. The results of the previous studies and the analysis reported here confirm that no two ELISAs give identical results with a specific panel of sera. Reference laboratory surveys generally give good agreement, ${ }^{4,6,16}$ but surveys involving diagnostic laboratories are often not as confirmatory. ${ }^{2,3}$

Although manufacturers can assist diagnostic laboratories by making their ELISAs as straightforward and as convenient to use as possible, ultimately the validity of the test result depends on the staff in the testing laboratory. In the study reported here, this variable was excluded by involving only very experienced staff in only one laboratory that has had a diagnostic and research commitment to toxoplasmosis over the last 15 years. These staff found that the Abbott-ELISA was by far the easiest of the ELISAs evaluated to perform, as there was no serum dilution step and reagents did not have to be reconstituted, diluted, or frozen for further use. The Sorin-ELISA, FMC-ELISA and REC-ELISA have acceptable methodology, and the Mercia-ELISA is very easy to use, the only reagents needing to be diluted being the washing buffer and chromogen.

Although the Abbott-ELISA was the easiest test to use, and was very reproducible with all coefficients of variation being $<7.3 \%$, its specificity was the lowest of the ELISAs tested. These results are similar to those found for this ELISA by Schaefer et al. ${ }^{5}$ The SorinELISA was considered to give overall satisfactory performance with regard to predictive values, specificity and sensitivity. The Mercia-ELISA stands out as being the only commercial ELISA that gave $100 \%$ negative predictive value and sensitivity. Furthermore, this ELISA showed a high positive pre- 
dictive value and a high specificity for serum panels in which the prevalence of $T$. gondii-specific antibodies was very high or very low, respectively. Considering this overall high performance and its ease of use, the Mercia-ELISA appears to be very suitable for largescale serological examinations, such as in seroepidemiological surveys.

Consistent with the results obtained with ELISAs based on H4/GST or H11/GST in a previous study, ${ }^{11}$ the REC-ELISA appeared to be the most specific ELISA in the comparison reported here, giving specificities and positive predictive values of $100 \%$ for all groups of sera tested. However, although negative predictive values reached $100 \%$ for serum panels with low prevalences of $T$. gondii-specific antibodies, the relatively low sensitivity of the REC-ELISA also resulted in a low overall negative predictive value. It is well-known from hybridoma technology that the use of highly specific monoclonal antibodies (MAbs) in immunological tests may cause problems with respect to sensitivity, due to the high selectivity and sometimes low avidity of these MAbs and the lack of co-operative effects between multiple types of antibodies in these test systems. ${ }^{17,18}$ Although we still know very little about the kinetics of recombinant antigen ELISAs, it can be expected that the use of highly specific recombinant antigens in immunodiagnostics may lead to similar phenomena as previously observed for MAbs. Srivastava et al. $^{19}$ found that the use of a mixture of three recombinant polypeptides of Plasmodium falciparum significantly increased the sensitivity of an ELISA, even when the single polypeptides were present at lower concentrations in the ELISA based on the mixture than in the ELISAs based on single recombinant polypeptides. Therefore, in the study reported here we used equal quantities of the two recombinant $T$. gondii polypeptides mixed together as an antigen preparation. Consistent with the findings of Srivastava et al. ${ }^{19}$ this resulted in an increase in sensitivity from $54 \%$ and $61 \%$ observed in our previous study ${ }^{11}$ for separate H4/GST and H11/GST, respectively, to $81.3 \%$ for the REC-ELISA described here. Antibody avidity increases with time after an antigenic challenge, and this fact has recently been used to diagnose acute toxoplasmosis via a low avidity IgG elution ELISA. ${ }^{20,21}$ Unlike the other ELISAs tested here, the REC-ELISA does not detect only $T$.

\section{References}

1. Dubey JP, Beattie CP. Toxoplasmosis of animals and man Boca Raton, FL, CRC Press. 1988: 41-48.

2. Johnson AM. Toxoplasmosis serology. The Royal College of Pathologists of Australasia Quality Assurance Programme Microbiology Survey 1990; M5: 7-8.

3. Johnson AM. Toxoplasmosis serology. The Royal College of Pathologists of Australasia Quality Assurance Program Microbiology Survey 1989; M6: 1-2.

4. Joynson DHM, Payne RA, Balfour AH, Prestage ES, Fleck DG, Chessum BS. Five commercial enzyme-linked immunosorbent assay kits for Toxoplasma specific IgM antibody. J Clin Pathol 1989; 42: 653-657.

5. Schaefer LE, Dyke JW, Meglio FD, Murray PR, Crafts W Niles AC. Evaluation of microparticle enzyme immuno- gondii-specific IgM, because it uses an anti-IgG (heavy and light chain) conjugate. ${ }^{11}$ Hence, the success of the REC-ELISA may rest on the avidity of the H4/GST and H11/GST fusion polypeptides with IgG or IgM, or both, antibodies to $T$. gondii produced during the acute stages of infection. The high coefficients of variation observed for the REC-ELISA in the analysis of within-assay precision with sera in which $T$. gondiispecific antibodies were not highly abundant may also be associated with the avidity of the antibodies directed to the unique antigens used in the REC-ELISA. However, the results obtained here suggest that the development of other types of recombinant $T$. gondii antigens and their addition to antigen preparations for use in ELISAs to diagnose toxoplasmosis will further enhance the sensitivity of these tests.

The REC-ELISA and the Abbott-ELISA are both indirect ELISAs, the FMC-ELISA and Sorin-ELISA are antibody class-capture ELISAs with unlabelled antigen, and the Mercia-ELISA is an antibody classcapture ELISA with labelled antigen. The indirect ELISA measures an absolute concentration of $T$. gondii-specific IgM, whereas the antibody classcapture system measures the immune load, which discriminates better between sera obtained during the early and later stages of toxoplasmosis., ${ }^{62} \mathrm{Un}$ fortunately we do not have sufficient clinical information on all patients, whose sera were used in this study to confirm or refute that this is the case, but there does not appear to be any obvious agreement of results with the type of ELISA performed.

All ELISAs tested in this survey gave satisfactory results. However, consistent with previous findings, no two tests agree totally, and it is up to the individual diagnostic laboratories that use the ELISA to establish the parameters of the test they choose to use in their own laboratory. Also, in this study we tested kits from only one batch from each manufacturer. Diagnostic laboratories using these ELISAs need to consider possible batch-to-batch variation.

We thank Mr D. Dickeson, Clinical Microbiology Unit, Department of Infectious Diseases and Microbiology, Westmead Hospital, NSW, for the provision of some discrepant sera, and Mr P. Phillips, Microbiology and Infectious Diseases, Flinders Medical Centre, for technical assistance. This work was carried out while A.M.T. was on leave from the Institut für Parasitologie der Tierärztlichen Hochschule Hannover, Germany, on a fellowship (Te 144/1-3) of the Deutsche Forschungsgemeinschaft

assays for immunoglobulins $\mathbf{G}$ and $\mathbf{M}$ to rubella virus and Toxoplasma gondii on the Abbott IMx automated analyzer. J Clin Microbiol 1989; 27 : 2410-2413.

6. Herbrink P, Van Loon AM, Rotmans JP, Van Knapen F, Van Dijk WC. Interlaboratory evaluation of indirect enzymelinked immunosorbent assay, antibody capture enzymelinked immunosorbent assay, and immunoblotting for detection of immunoglobulin $\mathrm{M}$ antibodies to Toxoplasma gondii. J Clin Microbiol 1987; 25: 100-105.

7. Van Enk RA, James KK, Thompson KD. Evaluation of three commercial enzyme immunoassays for Toxoplasma and cytomegalovirus antibodies. Am J Clin Pathol 1991; 95: 428-434.

8. Johnson AM, Illana S. Cloning of Toxoplasma gondii gene fragments encoding diagnostic antigens. Gene 1991; 99: $127-132$ 
9. Parker SJ, Smith FM, Johnson AM. Murine immune responses to recombinant Toxoplasma gondii antigens. $J$ Parasitol 1991; 77: 402-409.

10. Tenter AM, Vietmeyer C, Johnson AM. Development of ELISAs based on recombinant antigens for the detection of Toxoplasma gondii - specific antibodies in sheep and cats. Vet Parasitol 1992 (in press).

11. Tenter AM, Johnson AM. Recognition of recombinant Toxoplasma gondii antigens by human sera in an ELISA. Parasitol Res 1991; 77 : 197-203.

12. Naot Y, Barnett EV, Remington JS. Method for avoiding false-positive results occurring in immunoglobulin $M$ enzyme-linked immunosorbent assays due to presence of both rheumatoid factor and antinuclear antibodies. J Clin Microbiol 1981; 14: 73-78.

13. Johnson AM, Roberts H, McDonald PJ, Rothe J. Detection of Toxoplasma-specific IgM in cord blood sera by antibody. class capture enzyme-linked immunosorbent assay. Pathology 1985 ; 17 : 586-589.

14. Johnson AM, Gu QM, Roberts $H$. Antibody patterns in the serological diagnosis of acute lymphadenopathic toxoplasmosis. Aust NZ J Med 1987; 17: 430-434.

15. Griner PF, Mayewski RJ, Mushlin AI, Greenland P. Selection and interpretation of diagnostic tests and procedures. Ann Intern Med 1981; 94: 553-600.
16. Payne RA, Joynson DHM, Balfour AH et al. Public Health Laboratory Service enzyme-linked immunosorbent assay for detecting Toxoplasma specific IgM antibody. $J$ Clin Pathol 1987; 40: 276-281.

17. Campbell AM. General properties and applications of monoclonal antibodies. In: Burdon RH, Van Knippenberg PH (eds) Laboratory techniques in biochemistry and molecular biology, vol 13, Monoclonal antibody technology. Amsterdam, Elsevier Science Publishers. 1984: 1-32.

18. Mitchell GF. Hybridomas in immunoparasitology. In: Hurrell JGR (ed) Monoclonal hybridoma antibodies: techniques and applications. Boca Raton, CRC Press. 1982: 139-149.

19. Srivastava IK, Takacs B, Caspers $P$ et al. Recombinan polypeptides for serology of malaria. Trans $R$ Soc Trop Med Hyg 1989; 83: 317-321.

20. Hedman K, Lappalainen M, Seppälä I, Mäkelä O. Recent primary Toxoplasma infection indicated by a low avidity of specific IgG. J Infect Dis 1989; 159: 736-740.

21. Joynson DHM, Payne RA, Rawal BK. Potential role of IgG avidity for diagnosing toxoplasmosis. J Clin Pathol 1990; 43: 1032-1033.

22. Siegel JP, Remington JS. Comparison of methods for quantitating antigen-specific immunoglobulin $\mathbf{M}$ antibody with a reverse enzyme-linked immunosorbent assay. $J$ Clin Microbiol 1983; 18: 63-70. 